This document is the accepted manuscript version of the following article:

Hellmann, S., Calvi, M., Schmidt, T., \& Zhang, K. (2020). Numerical design optimization of short-period HTS staggered array undulators. IEEE Transactions on Applied Superconductivity, 30(4), 4100705 (5 pp.). https://doi.org/10.1109/TASC.2020.2965873

\title{
Numerical Design Optimization of Short-Period HTS Staggered Array Undulators
}

\author{
S. Hellmann, M. Calvi, T. Schmidt, K. Zhang
}

\begin{abstract}
Short period undulators are an essential component of a compact free electron laser (FEL) and medium energy storage rings for the production of hard X-rays. The use of $\mathrm{ReBCO}$ high temperature superconductors (HTS) in a staggered array undulator geometry is expected to yield a magnetic undulator flux density above $2 \mathrm{~T}$ for a $10 \mathrm{~mm}$ period and a $4 \mathrm{~mm}$ magnetic gap, thus substantially increasing the lasing performance of existing FEL and storage ring facilities.

The optimization of the design for a staggered array undulator with FEM simulations and a critical analysis of its working principles are discussed in this paper. Specifically, the maximization of the undulator field and the minimization of the required HTS material are the two main objectives of this study, which is performed with realistic material parameters of commercially available bulks materials.

Finally, the outcome of the applied optimization technique is discussed and the intrinsic limits of this undulator geometry are highlighted.
\end{abstract}

Index Terms - superconducting undulator, finite element method, $\boldsymbol{H}$-formulation, trapped current, trapped magnetic field, numerical optimization, $\operatorname{ReBCO}$ bulk material

\section{INTRODUCTION}

W ITH the use of modern high temperature superconducting materials, the performance of short period undulators for light source can be increased above what is so far possible with resistive conductors or permanent magnets. This has been predicted and demonstrated in the past by several research groups [1].

For undulators based on the concept of wound coils, the advantage of using superconducting wires or tapes simply arises from the significantly increased current density and the reduction in resistive losses. So far, these concepts were mainly demonstrated with the use of LTS wires $[2,3]$ whereas only a small number of research projects demonstrated undulator concepts with the use of HTS tapes and bulks [4, 5].

Apart from coil based superconducting undulators, the concept of the staggered array undulator stands out for its intriguing simplicity. This concept is based on staggered $R e B C O$ bulk pieces inside a solenoid surrounding the staggered array. For the undulator operation, the bulk pieces are gradually magnetized by changing the external field applied by the surrounding solenoid. This can be done using both, the field-cooling technique or the zero-field-cooling/pulsed-magnetization technique.

Since the current in the $R e \mathrm{BCO}$ bulk pieces can be controlled by the external solenoid field this design does not require current leads to be powered and the superconducting bulks pieces can be contained in a separate variable temperature insert (VTI) inside the solenoid. This can simplify the thermal concept, increase the cooling efficiency for the whole undulator setup and can further help to provide more stable operating temperatures for the HTS material.

This undulator concept, utilizing HTS material, was first proposed by Kii et. al. in 2006 [6] and is based on research work on conventional ferromagnetic staggered arrangements inside a solenoid [7]. Since the first proposal, additional work on the design and the predicted performance of such a device was published and first experimental tests were conducted [8, 9]. However, to the authors knowledge only a small amount of research was carried out on the design parameters for such a device [10] and no research work was published on the optimization of the design parameters and performance of a ReBCO bulk based staggered array undulator (thereafter referred to as staggered array undulator).

In this work we present the first numerical simulation method based on finite element analysis to find optimal geometrical parameters for a staggered array undulator design while optimizing for the resulting undulator field. The optimization is conducted for different assumed material characteristics of the $R e \mathrm{BCO}$ bulks as well as for different magnetization schemes.

\section{Simulation MODEL}

The base of the numerical parameter optimization reported in this paper is a transient 3D-FEM (finite element method) model applying the magnetic field formulation ( $H$-formulation) implemented into the commercial finite element simulation tool COMSOL Multiphysics ${ }^{\circledR}$. The $H$-formulation is widely used to

Color versions of one or more of the figures in this paper are available online at http://ieeexplore.ieee.org.

Digital Object Identifier will be inserted here upon acceptance.
This project has received funding from the European Union's Horizon 2020 research and innovation program under the Marie Skłodowska-Curie grant agreement No 701647.

S. Hellmann, M. Calvi, T. Schmidt and K. Zhang are with the Paul Scherrer Institute in Villigen, Switzerland (e-mail: sebastian.hellmann@psi.ch). 


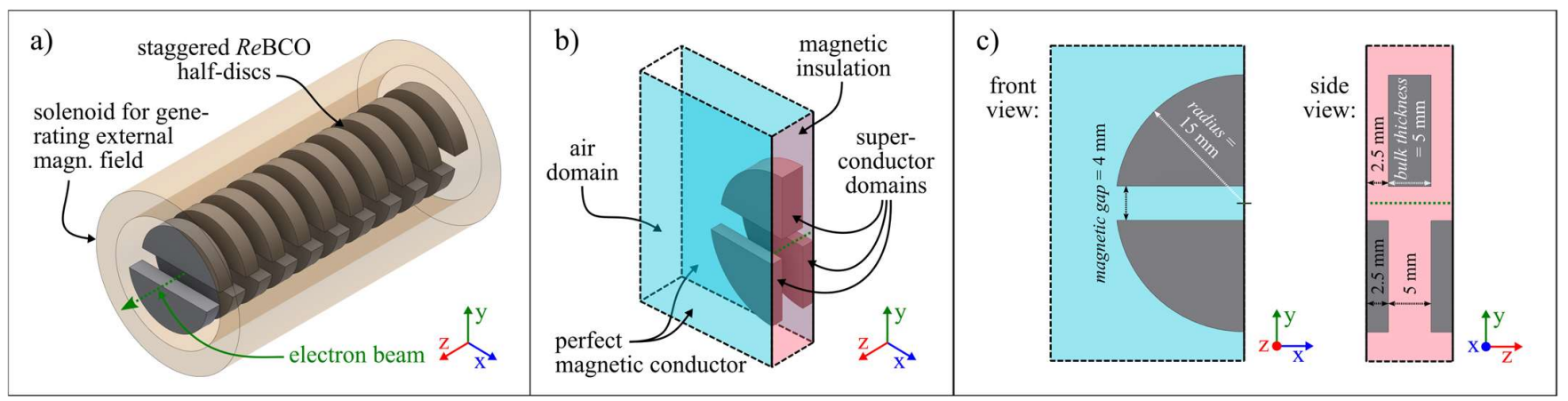

Fig. 1 a) Full geometry of a ten-period staggered array undulator consisting of 20 ReBCO half discs. In the application as an undulator in an electron accelerator the electron beam travels between the upper and the lower row of ReBCO bulks. b) Simulation model using two symmetries in the model. These are implemented by a magnetic insulation and perfect magnetic conductor boundary conditions. c) Dimensions of the reference undulator geometry and optimization parameters radius, bulk thickness and magnetic gap (the depicted sketches are not to scale)

solve transient Eddy-current and magnetization problems in superconductors $[11,12]$. The applied simulation model allows to simulate the magnetization procedure of a staggered array undulator as well as the expected undulator field under the assumption of a given set of geometric undulator parameters and material properties.

The geometry of the simulation model is presented in Fig. 1. Picture a) of Fig. 1 shows the full reference geometry of a tenperiod staggered array undulator inside a solenoid. To avoid unnecessary overhead in the simulation process, three symmetry planes are used to define a periodic single-period model (as shown in section b) of Fig. 1) instead of simulating a larger undulator geometry. The single-period model uses a magnetic insulation boundary condition to represent the vertical mirror plane along the electron beam and a perfect magnetic conductor boundary condition to represent the periodicity of the full staggered array undulator.

Section c) in Fig. 1 shows the dimensions of the reference geometry of the staggered array undulator. The reference geometry is used for comparison purposes during the optimization process if not stated otherwise.

The magnetic field produced by the solenoid is represented in the single-period model by adding an external magnetic field boundary condition to all external boundaries but the magnetic insulation boundary. During the simulation of the magnetization process the external magnetic flux density is decreased in

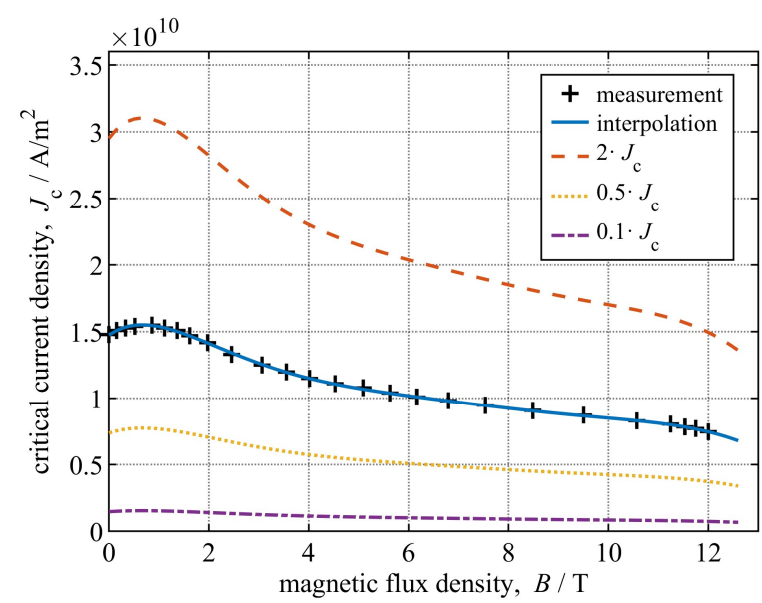

Fig. 2 Critical current density or ReBCO bulk material versus magnetic flux density at $4.2 \mathrm{~K}$. Additionally the graph depicts the scaled $J_{\mathrm{c}}(B)$-values

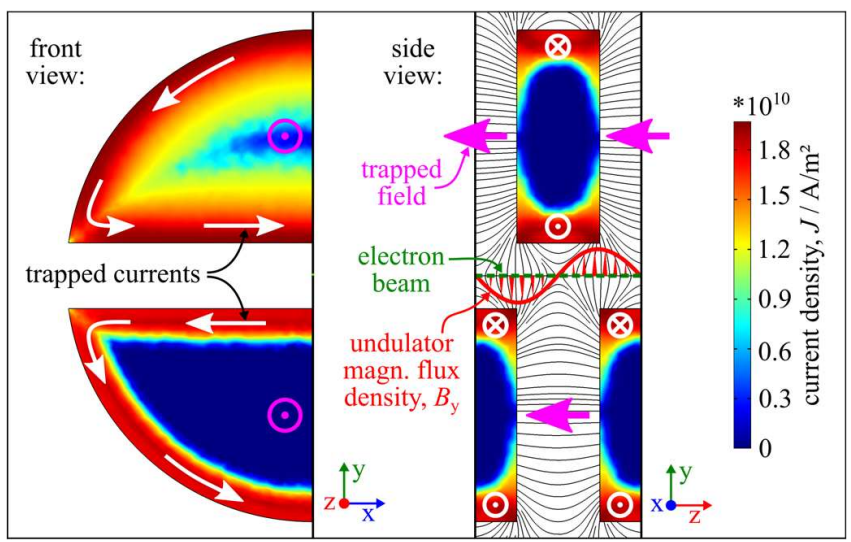

Fig. 3 Example of trapped currents and resulting trapped magnetic field and consequent undulator field after magnetization in the described transient numerical simulation

a linear ramp from $10 \mathrm{~T}$ to $0 \mathrm{~T}$ in in a specified magnetization time of $5 \mathrm{~s}$ or $1800 \mathrm{~s} .10 \mathrm{~T}$ in $1800 \mathrm{~s}$ or a corresponding field ramp of $<6 \mathrm{mT} / \mathrm{s}$ can be considered a realistic value for a modern superconducting magnet system of the $10 \mathrm{~T}$-class.

Since the external magnetic flux density of $10 \mathrm{~T}$ is applied as an initial condition of the transient model, this can be considered a field-cooling procedure.

The resistivity of the superconductor domains in the model follows a standard power law with an exponent of $n=20$.

The critical current density, used in the power law, is assumed to have a dependency on the magnetic flux density as shown in Fig. 2. However, the model does not include an additional dependency on the magnetic field angle due to the lack of available measurement data for ReBCO bulk material. The critical current density depicted in Fig. 2 was measured at $4.2 \mathrm{~K}$ with a magnetic field perpendicular to the $a b$-plane of the material.

Fig. 3 shows an example of a simulation result for the currents induced and trapped in the ReBCO bulks (white arrows) of the reference geometry after ramping the external magnetic flux density from $10 \mathrm{~T}$ to $0 \mathrm{~T}$ in $5 \mathrm{~s}$. The figure further depicts the main trapped field, as a result of the trapped currents, indicated by magenta arrows. It can be seen that the undulator field 
$B_{\mathrm{y}}$ in the staggered array undulator is created by the stray components of the main trapped field traversing between the upper and lower row of $\operatorname{ReBCO}$ bulks.

Fig. 3 further shows that the trapped currents exceed the critical current value by ca. $30 \%$. This extreme over-trapping of currents can be observed especially for short magnetization times and does not occur for longer ramping times.

\section{OPTIMIZATION PROCESS}

The optimization process described in this work is based on three different geometry parameter sweeps - over the radius, $r$ of the $R e$ BCO bulk pieces, the thickness, $t$ of the bulk pieces and over the gap, $g$ between the upper and the lower row of $R e$ BCO bulk pieces in the undulator geometry. During the parameter sweeps the peak value of the absolute undulator field, $\hat{B}_{y}$ along the electron beam path (shown as a dotted green line in Fig. 3) is evaluated for determining the value of the swept geometry parameter that yields the highest undulator field.

The parameter sweeps are performed independent of each other, meaning, that while one geometry parameter is altered the other parameters are kept at the standard value stated in the the reference undulator geometry (magnetic gap, $g=4 \mathrm{~mm}$, radius, $r=15 \mathrm{~mm}$ and bulk thickness, $t=5 \mathrm{~mm}$ ).

During one parameter sweep the described transient simulation model is solved repeatedly while changing the parameter under investigation.

The parameter sweep on one of the three geometry parameters is repeated for four scaling factors for the critical current density of the $\mathrm{ReBCO}$ material. This is done to represent a wider range of available $\operatorname{ReBCO}$ bulk materials. The scaling of the applied $J_{\mathrm{c}}(B)$ is done linearly with the factors $2,1,0.5$ and 0.1 .

The sweeps over the geometry parameter under investigation is also evaluated for different magnetization schemes. For the first two schemes the magnetization of the ReBCO bulks is simulated with a ramping time of $5 \mathrm{~s}$ and $1800 \mathrm{~s}$ respectively. The third magnetization scheme also uses a ramping time of $1800 \mathrm{~s}$ but in this case it is extended by an additional relaxation time of $1800 \mathrm{~s}$. During this relaxation time no external fields are applied to the model.

The magnetization state and consequent undulator field are always evaluated at the end of the simulation.

It should further be mentioned that a ramping time of $5 \mathrm{~s}$ is a pure hypothetical value which is not viable in an experiment and merely used in this optimization for comparison purposes.

\section{RESULTS AND DISCUSSION}

With the following graphs the results of the geometry parameter sweeps for the staggered array undulator geometry are presented. Each of the swept geometry parameters is depicted in a separate graph (Fig. 4, Fig. 6 and Fig. 5) and explained in the following subchapters.

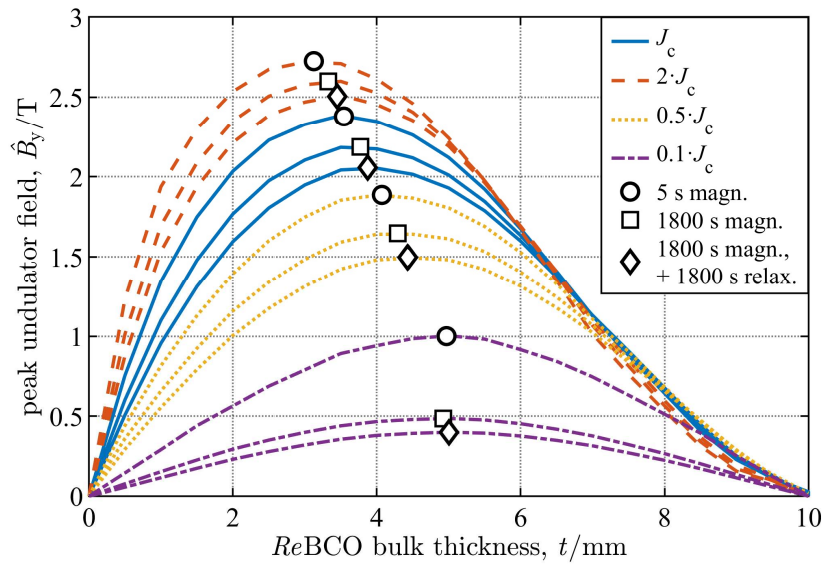

Fig. 4 Peak undulator field for the radius sweep over the thickness of the $R e B C O$ bulk pieces in the staggered array undulator geometry. The different line-styles represent different scaling factors for the critical current density while the different marker-styles represent different magnetization schemes applied in the transient simulation model. Additionally, the marker position in this plot represents the bulk radius yielding the highest peak undulator field

\section{A. ReBCO bulk thickness}

Fig. 4 shows the peak value of the absolute undulator field, $\hat{B}_{y}$ versus the bulk thickness.

The four different line-styles in the plot shown in Fig. 4 represent the sweeps with different applied scaling factors for the critical current density. The different markers identify the three magnetization schemes used for the corresponding parameter sweep. Further, in this graph, the position of the markers shows the bulk thickness yielding the highest resulting undulator field for each of the twelve sweeps. The exact location of the maximum value is calculated by fitting the individual curves with a higher degree polynomial function and determining the precise maximum location.

It can be seen that the undulator fields decreases to zero for both extreme values, a bulk thickness of $0 \mathrm{~mm}$ and a thickness of $10 \mathrm{~mm}$. Obviously for a thickness of $0 \mathrm{~mm}$, no ReBCO material is present in the model and therefore no undulator field is can be generate. For a thickness of $10 \mathrm{~mm}$ the upper and lower rows of $R e B C O$ bulks form a continuous row which does not allow any stray field traversing the gap and therefore, also in this case, the undulator field drops to zero.

The different resulting undulator field for the three applied magnetization schemes can be clearly seen in the deviation between the three lines of each line-style. The line with the highest undulator field values, marked by circular markers, represents the magnetization with a field ramp of $5 \mathrm{~s}$. In these cases the almost instantaneous magnetization leads to trapped currents in the $R e \mathrm{BCO}$ bulk material that exceed the critical current value by up to $50 \%$. This over-trapped current leads to higher values for the undulator field.

A more realistic magnetization with a field ramp of $1800 \mathrm{~s}$ in length is represented in the graph by the lines marked with the quadratic marker. Here, the trapped currents still exceed the critical current density but only by values below $15 \%$ above $J_{\mathrm{c}}$. 


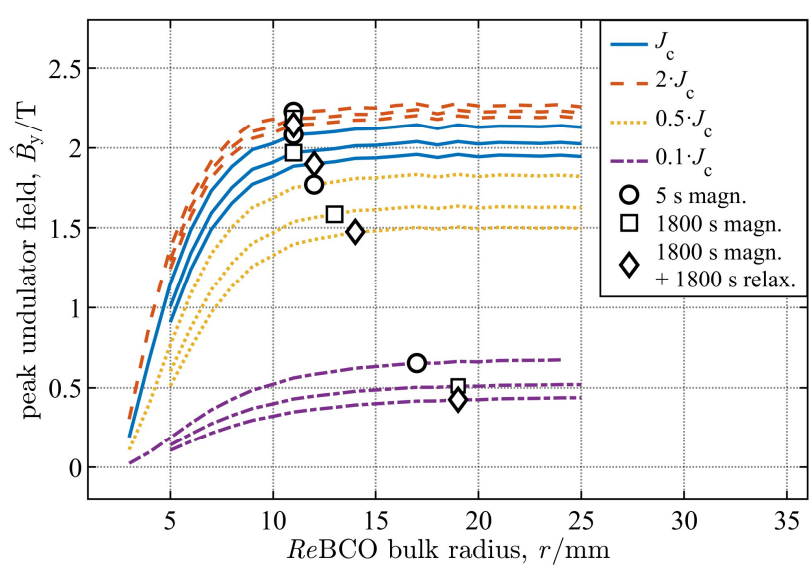

Fig. 5 The graph shows the peak undulator field versus the radius of the $R e \mathrm{BCO}$ bilk pieces in the staggered undulator geometry. The different linestyles represent different scaling factors for the critical current density value while the different marker-styles represent different magnetization schemes. The position of the markers indicate the minimum radius for achieving $97 \%$ of the undulator field at $25 \mathrm{~mm}$

The third magnetization scheme, with a ramping time of $1800 \mathrm{~s}$ followed by a relaxation time of $1800 \mathrm{~s}$, is shown in the graph by the lines marked with the diamond-shaped marker. After this additional relaxation time the trapped currents decrease to values around $95 \%$ of the critical current density value. This decay is the reason for the lower undulator field shown in the graph for the third magnetization schemes.

The graph further shows that for higher critical current densities values of the bulk material the optimum thickness shifts towards lower values and decreases down to $3.1 \mathrm{~mm}$ for $2 \cdot J_{\mathrm{c}}$. For lower critical current density values the maximum undulator field shifts towards higher values for the bulk thickness and settles around $5 \mathrm{~mm}$ for an assumed $10 \%$ of the reference $J_{\mathrm{c}}$.

It can also be seen in the graph that the ideal bulk thickness changes with the applied magnetization scheme.

\section{B. Radius of ReBCO half-discs}

Fig. 5 shows the results of the parameter sweeps over the radius of the ReBCO bulks. It can be seen that the peak undulator field increases from zero (for $r=g / 2$ ) towards higher values for the $R e \mathrm{BCO}$ bulk radius in an almost identical fashion for all scaled $J_{\mathrm{c}}$ values and magnetization scheme. This can be explained with the increasing amount of superconducting material that is available for magnetization and for carrying trapped current and field. The values for the maximum undulator field then converge towards a stable maximum value for larger bulk radii.

The position of the markers in this plot indicate the individual bulk radii which are necessary to yield $97 \%$ of the peak undulator field reached for the maximum tested radius of $25 \mathrm{~mm}$. This means, that a further increase of the radius beyond this point increases the undulator field only insignificantly because the $R e \mathrm{BCO}$ material is not fully penetrated by the induced current anymore. The position indicated by the markers can be seen as an optimized value for the bulk radius representing a compromise between the amount of $\mathrm{ReBCO}$ material required and the achieved peak undulator field.

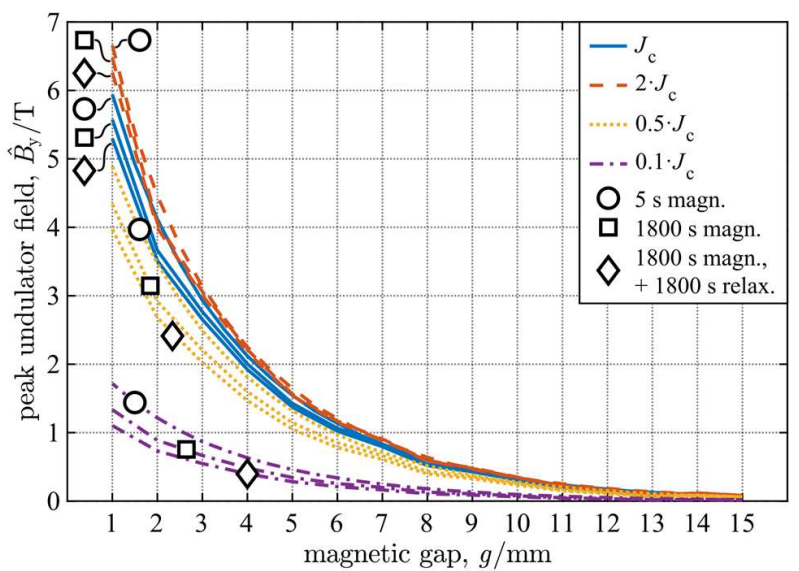

Fig. 6 Peak undulator field for changing the value for the magnetic gap between upper and lower row of $\operatorname{ReBCO}$ bulk pieces in the staggered undulator geometry. The different line-styles represent different scaling factors for the critical current density value while the different marker-styles represent different magnetization schemes. In this graph the marker position is of no meaning

It can further be seen that also in this geometry parameter sweep the magnetization scheme has a slight influence on the optimum radius.

\section{Magnetic Gap}

The graph in Fig. 6 illustrates the results of the parameter sweep over the magnetic gap between the upper and lower row of $R e \mathrm{BCO}$ bulk pieces in the staggered undulator geometry.

The parameter sweeps for all scaled critical current densities and magnetization schemes shows an exponential decrease of the undulator field for an increase of the magnetic gap size, $g$. This trend is to be expected since the magnetic field traversing between the upper and lower row of ReBCO bulks should decrease exponentially with the sources of the magnetic field (the trapped currents in the $R e \mathrm{BCO}$ bulks) moving away from each other and from the electron beam path.

Based on the nature of the dependency of the undulator field on the magnetic gap, this parameter sweep cannot yield a technically feasible optimum value. It rather gives an idea of the value of the undulator field that can be expected for a geometry with a chosen magnetic gap value as well as of the effect of mechanical tolerances on the undulator field. In a real-life undulator this magnetic gap value is usually as small as technically possible while still allowing enough room for necessary fixtures, tubing or vacuum chambers for the containment of the electron beam.

\section{CONCLUSION}

In this paper we present a way of determining the performance of a staggered array undulator depending on the variation of a set of geometry parameters as well as with different material parameters and magnetization schemes. The results of this study allow a deeper understanding of the complex correlation between the different parameters and the role they play in a staggered undulator geometry. This understanding is used 
to determine the optimum $R e \mathrm{BCO}$ bulk thickness, to find a suitable trade-off between the bulk radius and $R e B C O$ material used and to evaluate the undulator field that can be expected for a certain chosen magnetic gap value.

The presented results further show significant effects of flux creep in the HTS material after and during the magnetization process. This effect has to be addressed by future studies to allow a long-term stable and predictable operation of such a device in a storage ring facility or FEL.

\section{ACKNOWLEDGMENT}

The authors would like to thank Dr. Víctor Manuel Rodríguez Zermeño, Dr. Francesco Grilli and Dr. Mark Ainslie for many hours of very useful discussion about on the topic of the $H$-formulation and its versatile applications.

We would further like to thank the Adelwitz Technologiezentrum $\mathrm{GmbH}$ for providing us with the $J_{\mathrm{c}}(B)$ measurement data shown in Fig. 2.

\section{REFERENCES}

[1] Bahrdt, J. and E. Gluskin, Cryogenic permanent magnet and superconducting undulators. Nuclear Instruments and Methods in Physics Research Section A: Accelerators, Spectrometers, Detectors and Associated Equipment, 2018. 907: p. 149-168.

[2] Casalbuoni, S., et al., Characterization and long term operation of a novel superconducting undulator with $15 \mathrm{~mm}$ period length in a synchrotron light source. Physical Review Accelerators and Beams, 2016. 19(11).

[3] Schlueter, R., et al., Superconducting undulator research at LBNL. Synchrotron Radiation News, 2004. 17(1): p. 33-37.

[4] Kesgin, I., et al., High-temperature superconducting undulator magnets. Superconductor Science and Technology, 2017. 30(4).

[5] Tanaka, T., et al., Utilization of bulk high-temperature superconductors for shorter-period synchrotron radiation sources. Superconductor Science and Technology, 2006. 19(7): p. S438S442.

[6] Kii, T.Z., H.; Okawachi, N.; Nakano, M.; Masuda, K.; Ohgaki, H.; Yoshikawa, K.; Yamazaki, T. Design Study on High-Tc Superconducting Micro Undulator. in Proceedings of FEL 2006. 2006. Berlin, Germany.

[7] Ho, A.N.P., R.H.; Feinstein, J.; Huang,Y.C., A Solenoid-Derived Wiggler, IEEE Journal of Quantum electronics, 1991. 27(12).

[8] Kii, T., et al., Low-Temperature Operation of a Bulk HTSC Staggered Array Undulator. IEEE Transactions on Applied Superconductivity, 2012. 22(3): p. 4100904-4100904.

[9] Kinjo, R., et al., Demonstration of a High-Field Short-Period Undulator Using Bulk High-Temperature Superconductor. Applied Physics Express, 2013. 6(4).

[10] Kinjo, R., et al., Magnetic property of a staggered-array undulator using a bulk high-temperature superconductor. Physical Review Special Topics - Accelerators and Beams, 2014. 17(2)

[11] Ainslie, M.D. and H. Fujishiro, Modelling of bulk superconductor magnetization. Superconductor Science and Technology, 2015 28(5).

[12] Brambilla, R., F. Grilli, and L. Martini, Development of an edgeelement model for AC loss computation of high-temperature superconductors. Superconductor Science and Technology, 2007. 20(1): p. 16-24. 